\title{
Induction of Apoptosis and Growth Arrest in Human Breast Carcinoma Cells by a Snake (Walterinnesia aegyptia) Venom Combined With Silica Nanoparticles: Crosstalk Between Bcl2 and Caspase 3
}

\author{
Mohamed K. Al-Sadoon ${ }^{1} \quad$ Mostafa A. Abdel-Maksoud ${ }^{1}$ Danny M. Rabah' ${ }^{2,3}$ \\ Gamal Badr ${ }^{2,4}$ \\ ${ }^{1}$ Zoology Department, College of Science, King Saud University, Riyadh, Saudi Arabia; ${ }^{2}$ Princes Johara \\ Alibrahim Center for Cancer Research, Prostate Cancer Research Chair, College of Medicine, King Saud \\ University, Riyadh, Saudi Arabia; ${ }^{3}$ Department of Urology/Surgery, College of Medicine, King Saud \\ University, Riyadh, Saudi Arabia; ${ }^{4}$ Zoology Department, Faculty of Science, Assiut University, 71516 \\ Assiut, Egypt
}

\section{Key Words}

Apoptosis • Cytoskeleton • Nanoparticles $•$ Cell Signaling $•$ Snake Venom

\begin{abstract}
We recently demonstrated that the snake venom extracted from Walterinnesia aegyptia (WEV) either alone or combined with silica nanoparticles (WEV+NP) enhanced the proliferation of mice immune cells and simultaneously decreased the proliferation of human breast carcinoma cell line (MDA-MB-231). However, the molecular mechanism of how this venom induced growth arrest of breast cancer cells has not been studied. In this context, we extended our study to evaluate the anti-tumor potential of WEV and WEV+NP on the human breast carcinoma cell lines MDA-MB-231 and MCF-7, as well as their effects on non-tumorigenic normal breast epithelial cells (MCF-10). The IC ${ }_{50}$ values of WEV alone and WEV+NP in these cell lines were determined to be $50 \mathrm{ng} / \mathrm{ml}$ and $20 \mathrm{ng} / \mathrm{ml}$, respectively. Interestingly, at these concentrations, the venom did not affect the viability of normal MCF-10 cells and treatment of all these cell lines with NP alone did not affect their viability. Using annexin- $V$ binding assay followed by flow cytometry analysis, we found that combination of WEV with NP strongly induced apoptosis in MDA-MB-231 and MCF-7 cancer cells without significant effect on normal MCF-10 cells. Furthermore, we found that WEV+NP decreased the expression of $\mathrm{Bcl} 2$ and enhanced the activation of caspase 3 in MDA-MB-231 and MCF-7 cells. Most importantly, WEV+NP-treated breast cancer cells, but not normal MCF-10 cells, exhibited a significant $(P<0.05)$ reduction in actin polymerization and cytoskeletal rearrangement in response to CXCL12. Our data reveal biological effects of WEV or WEV+NP and the underlying mechanisms to fight breast cancer cells.




\section{Introduction}

Despite the major advances in the understanding of the mechanisms of breast cancer progression and in the development of novel therapeutic agents, breast cancer remains the second leading cause of mortality among women. Mortality is almost invariably due to metastasis $[1,2]$. Metastatic disease remains the most critical condition limiting patient survival, and the development of effective treatment against metastatic cancers, including breast cancer, is among the most important challenge in current experimental and clinical cancer research [3-5]. B cell CLL/Lymphoma 2 (Bcl2) family members are important regulators of cell survival and apoptosis in many cancers, including lung carcinoma, lymphoma and breast carcinoma [6, 7]. Of the Bcl2 family, Bcl2 is an anti-apoptotic protein that is frequently over expressed in several types of human cancers [8]. Over expression of anti-apoptotic members of the Bcl2 family such as Bcl2 and Bcl-xL has been implicated in cancer chemoresistance, whereas low levels of these proteins promote apoptosis and sensitize tumor cells to various anticancer therapies. Even though the mechanisms by which $\mathrm{Bcl} 2$ family proteins regulate apoptosis are diverse, ultimately they govern decision steps that determine whether certain caspase family members remain quiescent or become active $[9,10]$. Therefore, targeting the $\mathrm{Bcl} 2$ expression represents a promising strategy for the development of novel anti-cancer therapeutics. The therapeutic potential of targeting the Bcl2 signaling pathway is derived from the roles it plays in the promotion of cell growth and the inhibition of apoptosis. These properties are mediated by crosstalk between $\mathrm{Bcl} 2$ and several caspases that mediate apoptosis. Apoptosis is an essential and highly conserved mode of cell death that is important for normal development, host defense, and the suppression of oncogenesis. Apoptosis leads to removal of cancerous and virally infected cells, and the aberrant regulation of apoptosis is a major cause of tumor development and progression $[11,12]$. Caspase-3 is a major regulator of apoptosis [13].

Cell adhesion and motility are fundamental functions of both normal cells and metastatic tumor cells that involve both transmembrane adhesion receptors and intracellular signaling molecules $[14,15]$. The migration of cells through an extracellular matrix is a multistep process that begins with the extension of lamellipodia, cell-surface protrusions comprised of actin filaments, which are anchored to the underlying substratum by small, integrindependent focal adhesions [16]. In both normal and cancer cells, the polymerization of actin pushes against the plasma membrane and provides the force for forward movement. Within the cell body, actin stress fibers generate contractile forces by pulling against focal adhesions to induce retraction at the rear of the cell membrane. The bundling of actin filaments into stress fibers clusters and activates integrins, leading to the formation of new focal adhesions [17]. In normal cells, several transcription factors are activated following cell stimulation, leading to cytoskeletal rearrangement and proliferation. These signaling molecules modulate a wide range of intracellular events, including the regulation of actin polymerization, which is necessary for cells to change shape, form lamellipodia, and migrate $[18,19]$. Natural products have become increasingly important in pharmaceutical discoveries, and traditional herbalism has been a pioneering specialty in biomedical science. The search for effective natural anticancer agents has continued to gain momentum in recent years [20]. Several natural products have been used as anticancer agent inducing apoptosis and growth arrest of breast cancer cells. Recently, it has been shown that the kernel (Mangifera pajang) crude extract induced cytotoxicity in MCF-7 and MDA-MB-231 and that induced cycle arrest by activating caspase-2 and -3 [21]. It was found that Huaier extract could strongly inhibit viability of MCF-7 and MDA-MB-231 cells in a time- and dose-dependent manner through the activation of caspase-3 [22].

Snake venom is a complex mixture of many substances with a wide spectrum of biological activities including toxins, enzymes, growth factors, activators and inhibitors [23]. Natural toxins, especially a purified fraction of snake venom was used to reduce the size of the tumor and block angiogenesis [24]. Few data are available about the anti cancer effect of snake 
venom and the mechanisms through which snake venom may exert anti cancer properties. Therefore, in the present study, we investigated the effects of Walterinnesia aegyptia venom (WEV), alone and in combination with silica nanoparticles (WEV + NP) on the survival of 2 human breast carcinoma cell lines (MDA-MB-231 and MCF-7) and control non-tumorigenic normal breast epithelial cells (MCF-10) with special focus on the mechanisms through which it act as anti cancer agent.

\section{Materials and Methods}

Preparation of Walterinnesia aegyptia venom

Walterinnesia aegyptia. snakes were collected from the central region of Saudi Arabia. The snakes were kept in a serpentarium in the Zoology Department, College of Science, King Saud University. The snakes were warmed daily using a 100-watt lamp for nine hours, and water was always available. The snakes were fed purpose-bred mice every 10 to 14 days. The venom was milked from adult snakes, lyophilized and reconstituted in $1 \mathrm{X}$ phosphate-buffered saline (PBS) prior to use.

\section{Combination of snake venom with silica nanoparticles}

Silica nanoparticles and its combination with snake venom was prepared at King Abdullah Institute for Nanotechnology, King Saud University. Double mesoporous core-silica nanopsheres shell is formed around silica cores by using anionic surfactant where the solid silica core is turned to mesoporous one. Firstly, for the Synthesis of solid silica core $0.875 \mathrm{ml}$ of aqueous ammonia was added into a solution containing $18 \mathrm{ml}$ of ethanol and $2.6 \mathrm{ml}$ of deionized water followed by the addition of $1.5 \mathrm{ml}$ of Tetraethyl orthosilicate (TEOS) to the solution with vigorous stirring. Then the resulting mixture was heated at $30^{\circ} \mathrm{C}$ for $60 \mathrm{~min}$ then silica precipitate was collected by centrifugation and washed three times with water. The molar composition of the suspension was as follows: TEOS:EtOH: $\mathrm{NH}_{3}: \mathrm{H}_{2} \mathrm{O}=1: 46.9: 3.2: 20.5 .1: 37: 1.1: 2.7$

Secondly, for synthesis of mesoporous core-shell nanosphere by using anionic surfactant silica $\mathrm{SiO}_{2}$ particles were dispersed in $15 \mathrm{ml}$ of $\mathrm{H}_{2} \mathrm{O}$ by ultrasonication for $10 \mathrm{~min}$. For suppressing the agglomeration of silica cores $1 \mathrm{~g} / \mathrm{L}$ of polyvinylpyrrolidone, was added with continuous stirring for $60 \mathrm{~min}$. Thereafter, 0.1 $\mathrm{ml}, 0.2933 \mathrm{~g}$ (1 mmol) and $1.5 \mathrm{ml}$ of 3-aminopropyltrimethoxysilane (APMS), N-Lauroylsarcosine sodium (Sar-Na) and TEOS were added, respectively to the reaction mixture with subsequent stirring at $50^{\circ} \mathrm{C}$ for $2 \mathrm{~h}$. The final solid was recovered by centrifugation, washed with deionized water and dried in an oven at $60^{\circ} \mathrm{C}$ for 12 hours. Template removal was done by heat-treatment in an air stream at $550^{\circ} \mathrm{C}$ for 6 hours. The resulting molar ratio was TEOS: $\mathrm{H}_{2} \mathrm{O}$ : APMS: Sar-Na: HCl: PVP = 1:331.6: 0.08: 0.14: 0.06: 5x10-3. Then, a total of $25 \mathrm{mg}$ of mesoporous silica nanoparticles was added to a solution of $50 \mathrm{mg} / \mathrm{ml}$ venom in water. The suspension was stirred for 2 hours; the evaporation of water was prevented. The mesoporous silica nanoparticles loaded with venom were recovered using high-speed centrifugation and dried in a vacuum oven at $60^{\circ} \mathrm{C}$. Transmission Electron Microscopy (TEM) analysis was performed using a JEOL JSM-2100F Electron microscope (Japan) operated at $200 \mathrm{kV}$. Nitrogen sorption isotherms were measured at $77 \mathrm{~K}$ with a Quantachrome NOVA 4200 analyzer (USA). Before measurements, the samples were degassed in a vacuum at $200^{\circ} \mathrm{C}$ for at least 18 hours. The Brunauer-Emmett-Teller (BET) method was utilized to calculate the specific surface areas (SBET) using adsorption data in a relative pressure range from 0.05 to 0.35 . By using the Barrett-Joyner-Halenda $(\mathrm{BJH})$ model, the pore volumes and pore size distributions were derived from the adsorption branches of isotherms, and the total pore volumes $(V t)$ were estimated from the adsorbed amount at a relative pressure $P / P 0$ of 0.992 .

\section{Cell culture and reagents}

Human MDA-MB-231 and MCF-7 breast cancer cells as well as non-tumorigenic normal breast epithelial cells (MCF-10) were obtained from Dr. Douaa Sayed at Assiut University, Egypt and maintained in a culture medium consisting of MEM supplemented with $10 \%$ heat-inactivated fetal bovine serum (FBS, EuroClone, Life Science Division, Milan, Italy). The anti-proliferative effect of WEV and WEV+NP or NP alone on these three cell lines was determined using the 3-(4, 5-dimethylthiazol-2-yl)-2, 5 diphenyltetrazolium bromide (MTT) uptake method. The cells were plated at $1 \times 10^{6}$ cells $/ \mathrm{ml}$ in $2 \mathrm{ml}$ of culture medium in six- 
well Costar plates (Corning, Corning, NY). The cells were treated with different concentrations of WEV or $\mathrm{WEV}+\mathrm{NP}$ for $1,2,6,12,24$ or $48 \mathrm{~h}$, and cytotoxicity was expressed as a relative percentage of the OD values measured in the control (NP), WEV- and WEV+NP-treated cells. Morphological changes were observed after exposure to NP, WEV and WEV+NP using a phase-contrast inverse microscope (Olympus, Japan).

\section{Apoptosis detection and flow cytometry analysis}

Early apoptosis was measured using the Annexin V binding assay and flow cytometry analysis. Briefly, cells were treated or not with medium alone (0), NP, WEV or WEV+NP for overnight and then were washed and incubated in PBS containing 30\% heat-inactivated human $\mathrm{AB}$ serum at $4^{\circ} \mathrm{C}$ for 30 min prior to staining with Annexin V-FITC for $30 \mathrm{~min}$ at $4^{\circ} \mathrm{C}$ using a commercial kit according to manufacturer's instructions (BD Biosciences, France). The cells were washed twice with PBS and re-suspended in buffer solution (1 X $10^{6} \mathrm{cells} / \mathrm{ml}$ ). Stained cells were analyzed using a flow cytometer (BD FACSCalibur, San Jose, CA) within $1 \mathrm{~h}$ of staining, following manufacturer's protocol. The percentage of cells undergoing apoptosis was determined by calculating the Annexin $\mathrm{V}$ positive cells.

\section{Immunoblotting}

Whole-cell lysates were prepared from each cell line that were treated with or without NP, WEV- and WEV+NP in RIPA buffer (20 mM Tris- $\mathrm{HCl}, \mathrm{pH} 7.5,120 \mathrm{mM} \mathrm{NaCl}, 1.0 \%$ Triton X100, 0.1\% SDS, 1\% sodium deoxycholate, $10 \%$ glycerol, $1 \mathrm{mM}$ EDTA and $1 \%$ protease inhibitor cocktail, Roche). Following centrifugation at $16,000 \times g$ at $4^{\circ} \mathrm{C}$ for $15 \mathrm{~min}$, the protein concentrations in the supernatants were determined using a protein assay kit (Bio-Rad, Hercules, CA) and BCA protein assay method. Equal amounts of whole-cell protein $(50 \mu \mathrm{g})$ were mixed with reducing sample buffer $(0.92 \mathrm{M}$ Tris-HCl, pH 8.8, $1.5 \% \mathrm{SDS}$, 4\% glycerol, and $280 \mathrm{mM}$ 2-Mercapto-ethanol) and separated using discontinuous SDS-PAGE. Proteins were transferred onto nitrocellulose membranes using a Bio-Rad Trans-Blot electrophoretic transfer device, and the membranes were blocked for $1 \mathrm{~h}$ at room temperature with 1\% BSA or 5\% skim milk dissolved in TBS (20 mM Tris$\mathrm{HCl}, \mathrm{pH} 7.4$, and $150 \mathrm{mM} \mathrm{NaCl}$ ) supplemented with $0.1 \%$ Tween 20. The membranes were then incubated in the same blocking buffer with anti-Bcl2, anti-active caspase 3 or anti- $\beta$-actin antibodies $(1: 1,000$; Cell Signaling Technology, Beverly, MA). The blots were thoroughly rinsed and then incubated with an HRPlabeled species-matched secondary antibody for another $1 \mathrm{~h}$. Protei $\mathrm{n}$ bands were detected using enhanced chemiluminescence reagents (ECL, SuperSignal West Pico Chemiluminescent Substrate, Perbio, Bezons, France), and the ECL signals were recorded on Hyperfilm ECL. To quantify band intensities, the films were scanned, saved as TIFF files and analyzed using NIH ImageJ software.

\section{F-actin polymerization assay}

The three cell lines were cultured for 12 hours in culture medium supplemented with or without NP, WEV or WEV+NP before the F-actin polymerization test. Intracellular F-actin polymerization was assessed as previously described [25]. Briefly, cells were harvested and resuspended $\left(4 \times 10^{6} / \mathrm{ml}\right)$ in HEPESbuffered RPMI 1640 at $37^{\circ} \mathrm{C}$ with or without CXCL12 $(250 \mathrm{ng} / \mathrm{ml})$. At the indicated times, cell suspensions $(100 \mu \mathrm{l})$ were added to $400 \mu \mathrm{l}$ of assay buffer containing $4 \times 10^{-7} \mathrm{M}$ FITC-labeled phalloidin, $0.5 \mathrm{mg} / \mathrm{ml}$ L- $\alpha$-lysophosphatidylcholine (both from Sigma-Aldrich) and $4.5 \%$ formaldehyde in PBS. The fixed cells were analyzed using flow cytometry, and the mean fluorescence intensity (MFI) was determined for each sample. The percentage change in MFI was calculated for each sample at each time point using the following formula: 1-(MFI before the addition of CXCL12/MFI after the addition of CXCL12) $\times 100$.

\section{Statistical analyses}

Data were first tested for normality (using Anderson-Darling test) and for variances homogeneity prior to any further statistical analysis. Data were normally distributed and were expressed as the mean \pm standard error of the mean (SEM). Significant differences among groups were analyzed using a one-way analysis of variance (for more than two groups) followed by Tukey's post-test using SPSS software, version 17. Differences were considered statistically significant at $\mathrm{P}<0.05$. 

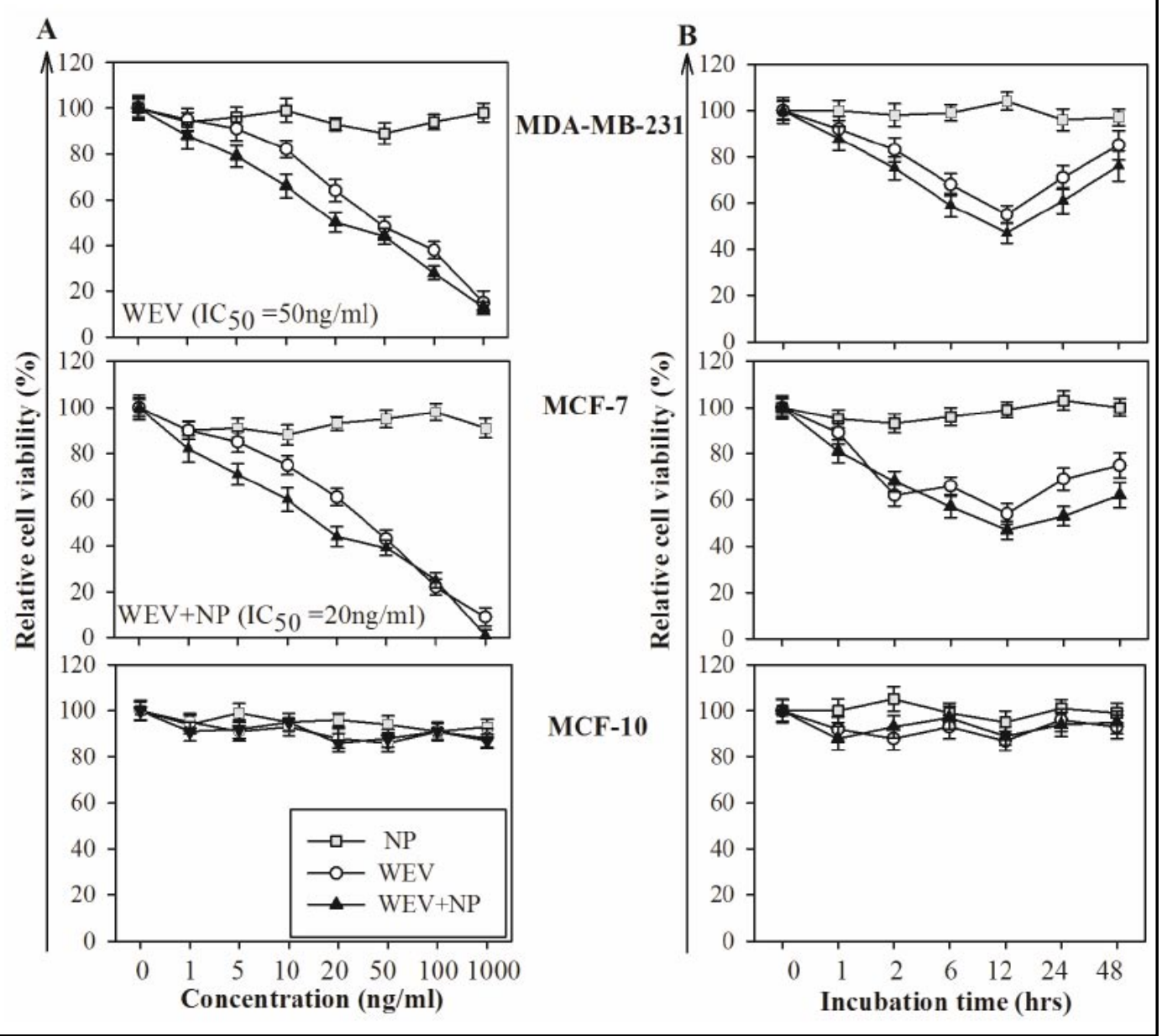

Fig. 1. Time- and dose-dependent responses of cell viability after WEV and WEV+NP treatment. MDA-MB231, MCF-7 and MCF-10 cells were treated for overnight with NP (open squares), WEV (open circles) or WEV+NP (closed triangles) at concentrations of 1, 5, 10, 20, 50, 100 and 1,000 ng/ml (A). The same cells were treated without (0) or with NP, WEV $(50 \mathrm{ng} / \mathrm{ml})$ and WEV+NP (20 ng/ml)for different incubation times $(1,2,6,12,24$ and $48 \mathrm{hr})(\mathrm{B})$. The experiments were performed in triplicate, and the results are expressed as the mean percentage of viable cells \pm SEM.

\section{Results}

WEV alone or combined with NP decrease the cell viability of breast cancer cells

The effect of WEV or WEV+NP on the viability of MDA-MB-231, MCF-7 and MCF-10 cells was assessed using the MTT uptake method. WEV inhibited the growth of MDA-MB-231 and MCF-7, but not of MCF-10 cells in a dose (Fig. 1 A)- and time (Fig. 1 B)-dependent manner. The combination of WEV with NP (WEV+NP) enhanced the effect of WEV on the cancer cells. The maximal inhibitory effects of WEV and WEV+NP on cell viability were observed 12 hours after treatment with $50 \mathrm{ng} / \mathrm{ml}$ of WEV alone or with $20 \mathrm{ng} / \mathrm{ml}$ of WEV+NP. Nevertheless, treatment WEV or WEV+NP had no significant inhibitory effect on the viability of nontumorigenic normal breast epithelial MCF-10 cells. These experiments were performed in triplicate, and the results are expressed as the mean percentage of viable cells \pm SEM. These results were confirmed using a Trypan blue exclusion test, which was used to determine cell viability and numerical cell counts simultaneously in all experiments (Table 1). 
WEV combined with NP induces apoptosis and growth arrest of breast cancer cells

Because treatment with WEV and WEV+NP decreased cell viability of cancer cells without affecting the viability of non-tumorigenic normal breast epithelial cells (MCF-10), we investigated whether treatment with WEV or WEV+NP decreased cell viability by induction of apoptosis or necrosis, a phenomenon important for the growth arrest of cancer cells. We detected, using annexin-V commercial kit and flow cytometry analysis, the percentage of cells undergoing apoptosis after treatment with NP alone, WEV, or WEV+NP. In one representative experiment (Fig. $2 \mathrm{~A}$ ) we found that the percentage of apoptotic cells was markedly increased after treatment with WEV in MDA-MB-231 and MCF-7 cells. Furthermore, treatment with 

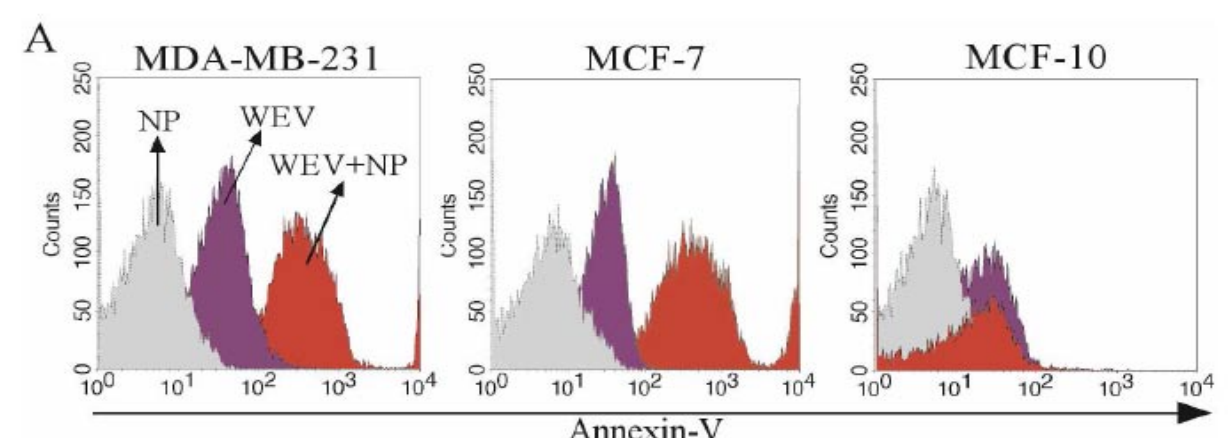

B

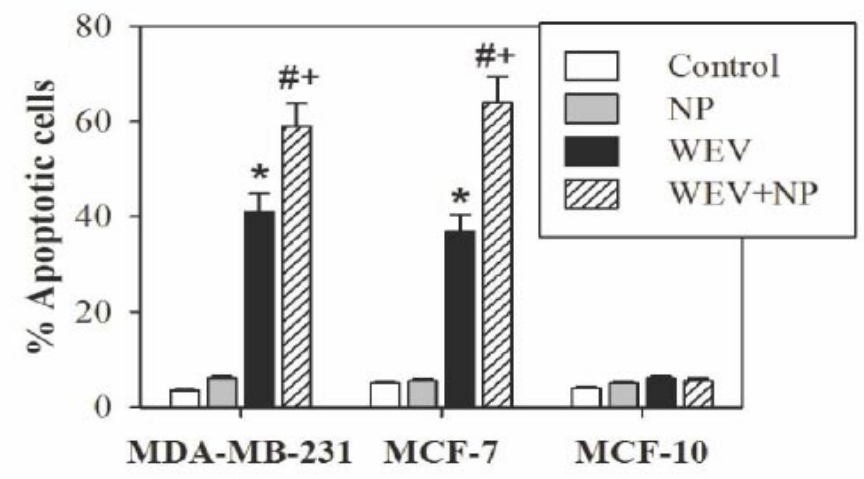

Fig. 2. WEV combined with NP induced apoptosis in breast cancer cells. The potential of WEV and WEV+NP to induce apoptosis was evaluated using annexin $\mathrm{V}$ binding assay and flow cytometry. The analysis of annexin $\mathrm{V}$ stained cells was performed after gating on the homogenous cell population. (A) One representative experiment of the five is shown for histograms of annexin V stained cells. (B) Data from five different experiments are expressed as the mean percentage of apoptotic cells \pm SEM for the control (open bars), NPtreated (gray bars), WEV-treated (closed black bars) and WEV+NP-treated (hatched bars). ${ }^{*} \mathrm{P}<0.05$, WEVtreated vs. NP; ${ }^{P}<<0.05, \mathrm{WEV}+\mathrm{NP}$-treated vs. NP; ${ }^{+} \mathrm{P}<0.05, \mathrm{WEV}+\mathrm{NP}$-treated vs. WEV-treated cells.

WEV+NP strongly enhanced apoptosis in breast cancer cells more than treatment with WEV alone.

Our results from five independent experiments (Fig. 2 B) revealed that the percentage of apoptotic cells was significantly increased, changing from $6 \pm 1 \%$ in the NP-treated MDAMB-231 cells to $41 \pm 4 \%$ and $59 \pm 5 \%$ in the WEV- and WEV+NP-treated MDA-MB-231 cells, respectively $\left({ }^{*} \mathrm{P}<0.05\right.$, WEV vs. control; ${ }^{\#} \mathrm{P}<0.05$, WEV+NP vs. control; ${ }^{+} \mathrm{P}<0.05$, WEV+NP vs. WEV; $n=5)$. Similarly, the percentage of apoptotic cells was significantly increased, changing from $5.5 \pm 0.5 \%$ in the NP-treated MCF- 7 cells to $37 \pm 3 \%$ and $64 \pm 5 \%$ in the WEV-and WEV+NPtreated MCF-7 cells respectively. Nevertheless, in non-tumorigenic MCF-10 treatment with WEV alone or WEV+NP did not significantly increase the percentage of apoptotic cells as compared to treatment with NP alone or with medium (0). In all experiments, treatment with NP alone did not significantly affect the percentage of apoptotic cells as compared to not treated cells.

WEV or WEV+NP mediated growth arrest of cancer cells is dependent on the down regulation of Bcl2 and up-regulation of active caspase 3

$\mathrm{Bcl} 2$ is considered as a prognostic marker that promotes long-term survival of breast cancer cells and functions to prevent apoptosis of these cancer cells in vivo and in vitro. We therefore monitored the impact of WEV and WEV+NP on the expression of Bcl2 in breast cancer cells and the crosstalk between $\mathrm{Bcl} 2$ and active caspase 3 as a pro-apoptotic protein in tumor progression. Using antibodies against $\mathrm{Bcl} 2$, active caspase 3 and $\beta$-actin (as a control for the amount of protein loaded) in western blot analyses, we normalized the levels 
Fig. 3. Effect of WEV and $\mathrm{WEV}+\mathrm{NP}$ on the expression of $\mathrm{Bcl} 2$ and active caspase 3 . The expression of $\mathrm{Bcl} 2$ and active caspase 3 was monitored in the three cell lines using western blot analysis. Protein bands from one representative experiment of the five performed are shown for the expression of $\mathrm{Bcl} 2$, active caspase 3 and $\beta$ actin in (A) MDA-MB-231, (B) MCF-7 and (C) MCF10 cells. The expression of $\mathrm{Bcl} 2$ and active caspase 3 was normalized to the total $\beta$-actin levels. The results are expressed as the mean \pm SEM of normalized values of Bcl2 (closed black bars) and active caspase 3 (open bars) in non treated cells (0), NP-, WEV-, and WEV+NPtreated cells. ${ }^{*} \mathrm{P}<0.05$, WEV-treated vs. NP; "P < $0.05, \mathrm{WEV}+\mathrm{NP}$-treated vs. $\mathrm{NP} ;{ }^{+} \mathrm{P}<0.05, \mathrm{WEV}+\mathrm{NP}-$ treated vs. WEV-treated cells.

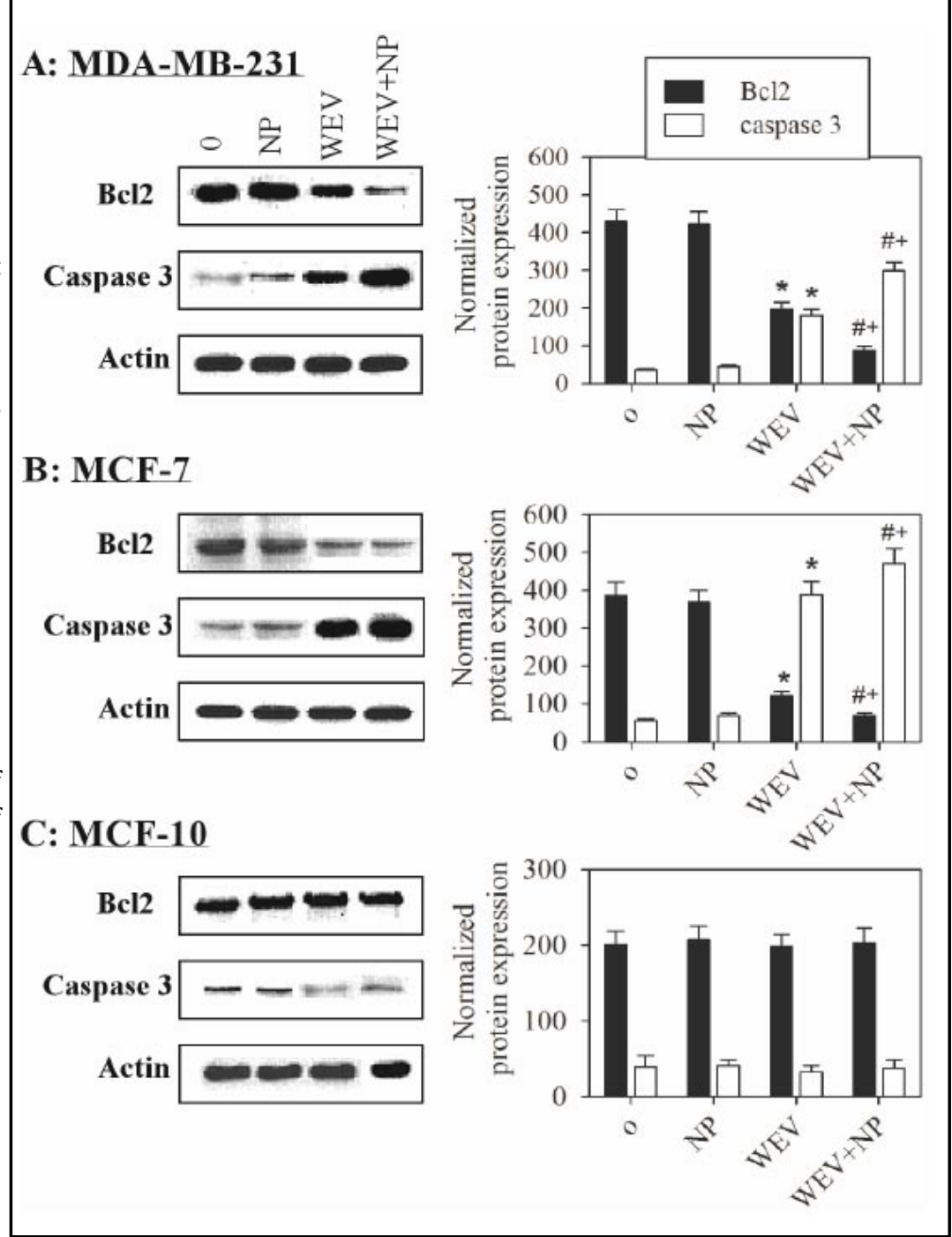

of $\mathrm{Bcl} 2$ and active caspase 3 to the amount of total $\beta$-actin. In one representative experiment (Fig. $3 \mathrm{~A}$ ), we observed that the expression of $\mathrm{Bcl} 2$ was obviously decreased in WEV- and WEV+NP-treated MDA-MB-231 and MCF-7 breast cancer cells, but not in non-tumorigenic MCF-10 cells. In contrast, the expression of active caspase 3 was inversely correlated with the expression of $\mathrm{Bcl} 2$ where its expression was clearly increased in WEV- and WEV+NPtreated MDA-MB-231 and MCF-7 breast cancer cells, but not in non-tumorigenic MCF-10 cells. Nevertheless, treatment with NP alone did not affect neither the expression of Bcl2 nor active caspase 3 in both MDA-MB-231 and MCF-7 breast cancer cells.

The results from five independent experiments (Fig. $3 \mathrm{~B}$ ) showed that the normalized levels of Bcl2 were significantly decreased from $422 \pm 32$ in NP-treated MDA-MB-231 cells to $197 \pm 19$ and $89 \pm 9$ in WEV- and WEV+NP-treated MDA-MB-231 cells respectively $\left({ }^{*} \mathrm{P}<0.05\right.$, WEV vs. control; ${ }^{\#} \mathrm{P}<0.05, \mathrm{WEV}+\mathrm{NP}$ vs. control; ${ }^{+} \mathrm{P}<0.05$, WEV+NP vs. WEV; $\mathrm{n}=5$ ). Similarly, the normalized levels of $\mathrm{Bcl} 2$ were significantly decreased from $370 \pm 31$ in NP-treated MCF7 cells to $121 \pm 11$ and $69 \pm 7$ in WEV- and WEV+NP-treated MCF-7 cells respectively ${ }^{*} \mathrm{P}<$ $0.05, \mathrm{WEV}$ vs. control; ${ }^{\#} \mathrm{P}<0.05, \mathrm{WEV}+\mathrm{NP}$ vs. control; ${ }^{+} \mathrm{P}<0.05$, WEV+NP vs. WEV; $\mathrm{n}=5$ ). In contrast, the normalized levels of active caspase 3 were significantly increased from $46 \pm 5$ in NP-treated MDA-MB-231 cells to $181 \pm 16$ and $298 \pm 23$ in WEV- and WEV+NP-treated MDAMB-231 cells respectively, and from $62 \pm 6$ in NP-treated MCF-7 cells to $389 \pm 35$ and 470 
Fig. 4. Effect of $W E V$ and $W E V+N P$ on the cytoskeleton rearrangement. MDA-MB-231 (A), MCF-7 (B) and MCF-10 (C) cells were treated for 12 hours with NP-treated (gray squares), WEV-treated (open circles) and WEV+NP-treated (closedtriangles).Cellswere then subjected to an F-actin polymerization assay after CXCL12 stimulation at the indicated time intervals (each 15 second), and the results were quantified using flow cytometry. The results are expressed as the percentage change in MFI $(n=5) \pm$ SEM.

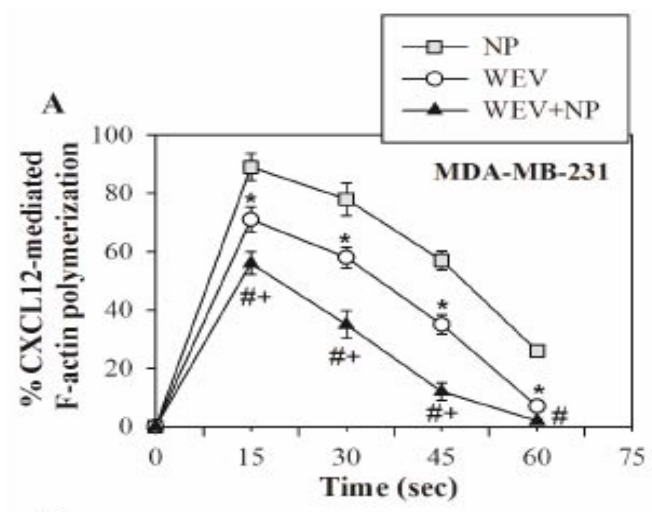

B
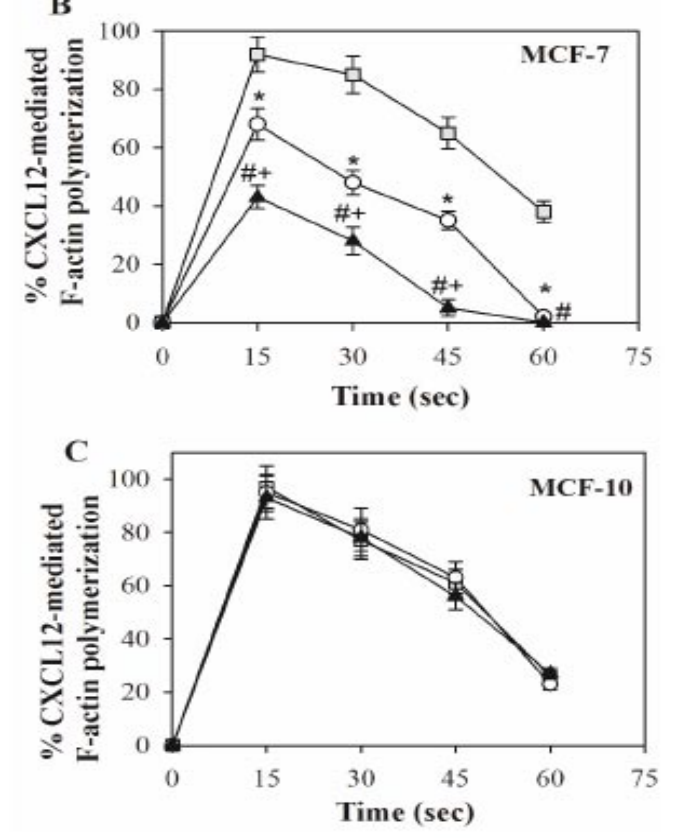

\pm 41 in WEV- and WEV+NP-treated MCF-7 cells respectively $\left({ }^{*} \mathrm{P}<0.05\right.$, WEV vs. control; ${ }^{*} \mathrm{P}<$ $0.05, \mathrm{WEV}+\mathrm{NP}$ vs. control; ${ }^{+} \mathrm{P}<0.05, \mathrm{WEV}+\mathrm{NP}$ vs. WEV; $\mathrm{n}=5$ ). Interestingly, neither treatment with WEV nor with WEV+NP affected significantly the expression of $\mathrm{Bcl} 2$ and active caspase 3 in non-tumorigenic MCF-10 cells. Additionally, in the three cell lines the normalized values of $\mathrm{Bcl} 2$ and active caspase 3 in NP-treated cells were nearly similar to those in non treated cells (0) demonstrating that treatment with NP alone did not affect the expression of Bcl2 or active caspase 3 .

\section{WEV combined with NP decreases CXCL12-mediated actin polymerization and cytoskeleton rearrangement}

Cytoskeletal organization plays a central role in cell movement, migration, adhesion, proliferation, differentiation, vesicle trafficking and survival in both normal and malignant cells. Actin and microtubules provide a dynamic cellular framework that orchestrates and ultimately controls cellular activation and cancer metastasis. We therefore investigated whether WEV alone or in combination with NP affected the actin polymerization induced by CXCL12, a chemokine that plays a critical role in the survival and metastasis of breast cancer cells. The cells were stimulated every $15 \mathrm{sec}$ with CXCL12 $(250 \mathrm{ng} / \mathrm{ml})$, and the degree of Factin polymerization was determined using F-actin assay and flow cytometry. Accumulated data from 5 independent experiments revealed that treatment with WEV (open circles) and $\mathrm{WEV}+\mathrm{NP}$ (closed triangles) significantly reduced the percentage of F-actin polymerization 
in MDA-MB-231 (Fig. 4 A) and MCF-7 cells (Fig. 4 B) as compared to NP-treated cells (gray squares). In contrast, treatment with WEV or WEV+NP did not significantly alter the percentage of F-actin polymerization induced by CXCL12 in non-tumorigenic MCF-10 cells (Fig. 4 C).

\section{Discussion}

The anti tumor and cytotoxic effects of snake venom on various types of cancer cells have been recently investigated $[26,27]$. However, little is known about its effects on breast cancer cells. We have recently demonstrated that the snake venom extracted from Walterinnesia aegyptia (WEV) either alone or combined with silica nanoparticles (WEV+NP) enhanced the proliferation of mice immune cells and simultaneously decreased the proliferation of human breast carcinoma cell line (MDA-MB-231) [28]. However, the molecular mechanism of how this venom induced growth arrest of breast cancer cells has not been studied. Here, we investigated the anti-tumor potential of WEV either alone or combined with silica NP on the human breast carcinoma cell lines MDA-MB-231 and MCF-7, as well as their effects on non-tumorigenic normal breast epithelial cells (MCF-10). The expression of cell signaling proteins involved in cell apoptosis, proliferation, and survival is a critically important event during metastatic spreading [29], so it was the main focus of the current study. First, we assessed the ability of WEV alone or combined with NP to arrest the growth of MDA-MB231, MCF-7 and MCF-10 cell lines, and we found that WEV affected the cell viability of breast cancer cells without inhibiting the viability of non-tumorigenic normal MCF-10 cells. The combination of WEV with NP (WEV+NP) markedly enhanced the inhibitory effect of WEV on the cancer cells.

Using nanoparticles to deliver chemotherapeutics offers new opportunities for cancer therapy. Therefore, the integration of drug delivery with nanotechnology in the field of cancer therapy has attracted much attention in recent decades [30, 31]. By improving drug bioaccessibility and chemosensitivity, nanoparticles as drug-delivery systems could increase therapeutic efficacy to fight cancer cells and mitigate side effects on normal surrounding noncancerous cells $[32,33]$. Subsequently, combination of WEV with nanoparticles increased the efficiency of WEV to arrest the growth of breast cancer cells without affecting the viability of non-tumorigenic normal MCF-10 cells. Since the MTT assay shows metabolic changes without absolute cell count, we investigated, using annexin-V commercial kit and flow cytometry analysis, the percentage cells undergoing apoptosis after treatment with WEV or WEV+NP. The percentage of apoptotic cells was increased after treatment with WEV in MDA-MB-231 and MCF-7 cells. Furthermore, treatment with WEV+NP strongly enhanced apoptosis in breast cancer cells more than treatment with WEV alone. Our results agree with the results of Son et al. [34] and Park et al. [27], who attributed this growth inhibition to apoptosis and cell cycle arrest. We next investigated the mechanism of cell cycle arrest exerted by this venom. It has been established that the crosstalk between $\mathrm{Bcl} 2$ family members control survival, tumor progression and resistance to chemotherapy in many cancers. Bcl2 is a well known anti-apoptotic protein that is frequently over expressed in several types of human cancers [8]. In contrast, caspase 3 is a well known mediator of apoptotic cell death [35]. Therefore, we monitored the impact of WEV and WEV+NP on the expression of Bcl2 in the breast cancer cells and the crosstalk between $\mathrm{Bcl} 2$ and active caspase 3 as a pro-apoptotic protein in tumor progression. The expression of Bcl2 was obviously decreased in WEV- and WEV+NP-treated MDA-MB-231 and MCF-7 breast cancer cells, but not in non-tumorigenic MCF-10 cells. In contrast, the expression of active caspase 3 was clearly increased in WEV- and WEV+NP-treated MDA-MB-231 and MCF-7 breast cancer cells, but not in non-tumorigenic MCF-10 cells. Nevertheless, treatment with NP alone did not affect neither the expression of Bcl2 nor active caspase 3 in both MDA-MB-231 and MCF-7 breast cancer cells. Similarly, Zhang et al. suggested that Husaier extract could strongly inhibit cell viability of MCF-7 and MDA-MB-231 cells in a time- and dose-dependent manner [22]. Induction of cell-cycle arrest 
in breast cancer cell lines through caspase-dependent apoptosis by natural compounds was also recently reported [35].

Actin cytoskeletal reorganization is the primary mechanism of cell motility and adhesion $[25,36]$. Actin-dependentcellular processes aretypically associated with membrane dynamics, and the coordinated polymerization of actin filaments against cellular membranes provides the force for these processes. Probably the most thoroughly characterized of such processes are cell migration towards chemokines. Therefore, we monitored actin polymerization in response to CXCL12 stimulation because both MDA-MB-231 and MCF-7 cells express CXCR4 and CXCR7 (data not shown), two chemokine receptors that bind to CXCL12. Similarly, Oliva et al. [37] suggested that RGD disintegrins isolated from snake venom were potent antimetastatic agents that contributed to the inhibition of melanoma cell invasion through the involvement of the actin cytoskeleton. The control of microfilament actin remodeling thus represents a potential target for the development of anticancer drugs [38]. Taken together, our data demonstrate the effect of snake venom as an anti-cancer agent. Most importantly, combination of WEV with nanoparticles strongly enhances the growth arrest of breast cancer cells through a direct effect on the crosstalk between $\mathrm{Bcl} 2$ and caspase 3 .

\section{Competing interests}

All authors have read and agreed the contents of the manuscript and approved the submission. The authors declare no conflicts of interest, state that the manuscript has not been published or submitted elsewhere, state that the work complies with Ethical Policies of the Journal and the work has been conducted under internationally accepted ethical standards after relevant ethical review.

\section{Abbreviations}

Bcl2 (B-cell CCL/Lymphoma 2); CXCL12 (CXC chemokine ligand 12); NP (nanoparticles); WEV (Walterinnesia aegyptia venom); WEV+NP (Walterinnesia aegyptia venom combined with nanoparticles).

\section{Acknowledgements}

This work was supported by the National Plan for Science and Technology (NPST) funded by King Abdulaziz City for Science and Technology (KACST) through project number 10-BI0969-02. The author also acknowledge Dr Ahmed El-Toni at the King Abdullah Institute for Nanotechnology, King Saud University, for loading the venom on the silica nanoparticles. The authors declare that they have no conflicts of interest.

\section{References}

1 Edlich RF, Winters KL, Lin KY: Breast cancer and ovarian cancer genetics. J Long Term Eff Med Implants 2005;15:533-545.

2 Lorusso G, Rüegg C: New insights into the mechanisms of organ-specific breast cancer metastasis. Semin Cancer Biol 2012;22:226-233.

3 Arai M, Taki K, Iwase H, Takizawa K, Nishimura S: Present status and tasks for genetic testing and riskreducing surgery in patients with hereditary breast and ovarian cancer. Gan To Kagaku Ryoho 2012;39:525531. 
Al-Sadoon/Abdel-Maksoud/Rabah/Badr: A Snake Venom Induces Apoptosis of Human Breast Cancer Cells

4 Brown S, Khan DR: The treatment of breast cancer using liposome technology. J Drug Deliv 2012;2012:212965.

5 Kim YH, Lee BJ, Lee KJ, Cho JH: A case of pituitary metastasis from breast cancer that presented as left visual disturbance. J Korean Neurosurg Soc 2012;51:94-97.

6 Andrews GA, Xi S, Pomerantz RG, Lin CJ, Gooding WE, Wentzel AL, Wu L, Sidransky D, Grandis JR: Mutation of P53 in head and neck squamous cell carcinoma correlates with BCL2 expression and increased susceptibility to cisplatininduced apoptosis. Head Neck 2004;26:870-877.

7 Kouri FM, Jensen SA, Stegh AH: The role of Bcl2 family proteins in therapy responses of malignant astrocytic gliomas: Bcl2L12 and beyond. ScientificWorldJournal 2012;2012:838916.

$>8$ Gao Q, Yang S, Kang MQ: Influence of survivin and Bcl2 expression on the biological behavior of non-small cell lung cancer. Mol Med Report2012;5:1409-1414.

9 Duo J, Ying GG, Wang GW, Zhang L: Quercetin inhibits human breast cancer cell proliferation and induces apoptosis via Bcl2 and Bax regulation. Mol Med Report 2012;5:1453-1456.

10 Larsen MS, Bjerre K, Giobbie-Hurder A, Lænkholm AV, Henriksen KL, Ejlertsen B, Lykkesfeldt AE, Rasmussen BB: Prognostic value of Bcl2 in two independent populations of estrogen receptor positive breast cancer patients treated with adjuvant endocrine therapy. Acta Oncol 2012;51:781-789.

11 Wen P Y, Kesari S: Malignant gliomas in adults. N Engl J Med 2008;359:492-507.

12 Seo HS, Choi HS, Kim SR, Choi YK, Woo SM, Shin I, Woo JK, Park SY, Shin YC, Ko SK: Apigenin induces apoptosis via extrinsic pathway, inducing p53 and inhibiting STAT3 and NFKB signaling in HER2-overexpressing breast cancer cells. Mol Cell Biochem 2012;366:319-334.

13 Boulares AH, Yakovlev AG, Ivanova V, Stoica BA, Wang G, Iyer S, Smulson M : Role of poly(ADP-ribose) polymerase (PARP) cleavage in apoptosis. Caspase 3-resistant PARP mutant increases rates of apoptosis in transfected cells. J Biol Chem 1999;274:22932-22940.

14 Kedrin D, van Rheenen J, Hernandez L, Condeelis J, Segall JE: Cell motility and cytoskeletal regulation in invasion and metastasis. J Mammary Gland Biol Neoplasia 2007;12:143-152.

15 Urra H, Torres VA, Ortiz RJ, Lobos L, Díaz MI, Díaz N, Härtel S, Leyton L, Quest AF: Caveolin-1-enhanced motility and focal adhesion turnover require tyrosine-14 but not accumulation to the rear in metastatic cancer cells. PLoS One 2012; 7:e33085.

16 Le Clainche C, Carlier MF: Regulation of actin assembly associated with protrusion and adhesion in cell migration. Physiol Rev 2008;88:489-513.

17 Wozniak MA, Modzelewska K, Kwong L, Keely PJ: Focal adhesion regulation of cell behavior. Biochim Biophys Acta 2004;1692:103-119.

18 Pollard TD: The cytoskeleton cellular motility and the reductionist agenda. Nature 2003;422:741-745.

19 Liu J, Zhang D, Luo W, Yu J, Li J, Yu Y, Zhang X, Chen J, Wu XR, Huang C: E3 Ligase Activity of XIAP RING Domain Is Required for XIAP-Mediated Cancer Cell Migration, but Not for Its RhoGDI Binding Activity. PLoS One 2012;7:e35682.

20 Shafi G, Hasan TN, Syed NA, Al-Hazzani AA, Alshatwi AA, Jyothi A, Munshi A: Artemisia absinthium (AA): a novel potential complementary and alternative medicine for breast cancer. Mol Biol Rep 2012;39:73737379.

21 Abu Bakar MF, Mohamad M, Rahmat A, Burr SA, Fry JR: Cytotoxicity, cell cycle arrest, and apoptosis in breast cancer cell lines exposed to an extract of the seed kernel of Mangifera pajang (bambangan). Food Chem Toxicol 2010;48:1688-1697.

22 Zhang N, Kong X, Yan S, Yuan C, Yang Q: Huaier aqueous extract inhibits proliferation of breast cancer cells by inducing apoptosis. Cancer Sci 2010;101:2375-2383.

23 El Hakim AE, Gamal-Eldeen AM, Shahein YE, Mansour NM, Wahby AF, Abouelella AM: Purification and characterization of a cytotoxic neurotoxin-like protein from Naja haje haje venom that induces mitochondrial apoptosis pathway. Arch Toxicol 2011;85:941-952.

24 Francis S, Markland A: Novel S nake venom disintegrin that inhibits human ovarian cancer dissemination and angiogenesis in an orthotopic nude mouse model. Haemostasis 2001;31:183-191.

25 Badr G, Borhis G, Treton D, Moog C, Garraud O, Richard Y: HIV type 1 glycoprotein 120 inhibits human B cell chemotaxis to CXC chemokine ligand (CXCL) 12, CC chemokine ligand (CCL) 20, and CCL21. J Immunol 2005;175:302-310. 


\section{Cellular Physiology and Biochemistry}

-26 Alama A, Bruzzo C, Cavalieri Z, Forlani A, Utkin Y, Casciano I, Romani M: Inhibition of the nicotinic acetylcholine receptors by cobra venom $\alpha$-neurotoxins: is there a perspective in lung cancer treatment? PLoS One 2011;6: e20695.

27 Park MH, Son DJ, Kwak DH, Song HS, Oh KW, Yoo HS, Lee YM, Song MJ, Hong JT: Snake venom toxin inhibits cell growth through induction of apoptosis in neuroblastoma cells. Arch Pharm Res 2009;32:1545-1554.

28 Badr G, Al-Sadoon MK, El-Toni AM, Daghestani M: Walterinnesia aegyptia venom combined with silica nanoparticles enhances the functioning of normal lymphocytes through PI3K/AKT, NFKB and ERK signaling. Lipids Health Dis 2012;15:11-27.

-29 Rajah TT, Peine KJ, DU N, Serret CA, Drews NR: Physiological Concentrations of Genistein and 17 $\beta$-Estradiol Inhibit MDA-MB-231 Breast Cancer Cell Growth by Increasing BAX/BCL2 and Reducing pERK1/2. Anticancer Res 2012;32:1181-1191.

-30 Allen TM, Cullis PR: Drug delivery systems: Entering the mainstream. Science 2004;303:1818-1822.

-31 Langer R, Tirrell DA: Designing materials for biology and medicine. Nature 2004;428:487-492.

-32 Davis ME, Chen Z, Shin DM: Nanoparticle therapeutics: An emerging treatment modality for cancer. Nat Rev Drug Disc 2008;7:771-782.

33 Moorthi C, Manavalan R, Kathiresan K: Nanotherapeutics to overcome conventional cancer chemotherapy limitations. J Pharm Pharm Sci 2011;14:67-77.

-34 Son DJ, Park MH, Chae SJ, Moon SO, Lee JW, Song HS, Moon DC, Kang SS, Kwon YE, Hong JT: Inhibitory effect of snake venom toxin from Vipera lebetina turanica on hormone-refractory human prostate cancer cell growth: induction of apoptosis through inactivation of nuclear factor kappaB. Mol Cancer Ther 2007;6:675-683.

35 Jung S, Li C, Lee S, Ohk J, Kim SK, Lee MS, Moon HI: Inhibitory effect and mechanism on antiproliferation of khellactone derivatives from herbal suitable for medical or food uses. Food Chem Toxicol 2012;50:648-652.

-36 Yamazaki D, Kurisu S, Takenawa T: Regulation of cancer cell motility through actin reorganization. Cancer Sci 2005;96:379-386.

-37 Oliva IB, Coelho RM, Barcellos GG, Saldanha-Gama R,Wermelinger LS, Marcinkiewicz C, Benedeta Zingali R, Barja-Fidalgo C: Effect of RGDdisintegrins on melanoma cell growth and metastasis: involvement of the actin cytoskeleton, FAK and c-Fos. Toxicon 2007;50:1053-1063.

-38 Rao J, Li N: Microfilament actin remodeling as a potential target for cancer drug development. Curr Cancer Drug Targets 2004;4:345-354. 